\title{
Lyman series emission after valence and core excitation of water vapor
}

\author{
A. Hans,${ }^{1, *}$ A. Knie,${ }^{1}$ Ph. Schmidt,${ }^{1}$ L. Ben Ltaief,${ }^{1}$ C. Ozga,${ }^{1}$ Ph. Rei,${ }^{1}$ \\ H. Huckfeldt, ${ }^{1}$ M. Förstel,${ }^{2}{ }^{\dagger}$ U. Hergenhahn, ${ }^{2}$ and A. Ehresmann ${ }^{1}$ \\ ${ }^{1}$ Institut für Physik, Universität Kassel, \\ Heinrich-Plett-Str. 40, 34132 Kassel, Germany \\ ${ }^{2}$ Max-Planck-Institut für Plasmaphysik, \\ Wendelsteinstr. 1, 17491 Greifswald, Germany
}

\begin{abstract}
We report Lyman series emission cross sections of neutral hydrogen dissociation fragments after valence $(15-34 \mathrm{eV})$ and inner-shell $(533-542 \mathrm{eV})$ excitation of water vapor with monochromatic synchrotron radiation as functions of the exciting-photon energy. In the valence excitation energy region the thermodynamical limits of the production of the differently excited hydrogen fragments is directly observed and absolute emission cross sections were determined. For resonant innershell excitations, the fluorescing excited hydrogen state is found to be strongly dependent on the molecular or Rydberg-like character of the excitation.
\end{abstract}

PACS numbers: 33.20.Ni, 33.50.-j, 33.70.Ca

\footnotetext{
*hans@physik.uni-kassel.de

$\dagger$ Now at: Department of Chemistry, University of Hawaii at Manoa, Honolulu, Hawaii, HI, 96822, USA
} 


\section{INTRODUCTION}

Because of its unique role in nature, the structure and dynamics of the water molecule has always been intensely investigated in molecular physics. Processes following photon irradiation are of particular interest in atmospheric physics and astrophysics, radiation biology, and fundamental photochemistry [1]. Depending on the incident photon energy, photodissociation, photoexcitation, or photoionization may occur, the latter two processes possibly followed or accompanied by photodissociation into neutral or ionic fragments. Recent progress in experimental techniques on liquid jets in high vacuum $[2,3]$ and theoretical and experimental investigations on water clusters [4-8] call for a complete understanding of the decay dynamics of the isolated molecule.

The electronic configuration of the water molecule in its ground state is

$$
1 \mathrm{a}_{1}^{2} 2 \mathrm{a}_{1}^{2} 1 \mathrm{~b}_{2}^{2} 3 \mathrm{a}_{1}^{2} 1 \mathrm{~b}_{1}^{2}\left({ }^{1} \mathrm{~A}_{1}\right)
$$

with an outer-valence ionization potential (of the $1 b_{1}$ orbital) of $12.62 \mathrm{eV}$ and binding energies of the inner-valence orbitals $2 \mathrm{a}_{1}, 1 \mathrm{~b}_{2}$, and $3 \mathrm{a}_{1}$ of $32.6 \mathrm{eV}, 18.6 \mathrm{eV}$, and $14.8 \mathrm{eV}$, respectively [9]. The core orbital $1 \mathrm{a}_{1}$ is basically of $\mathrm{O} 1 \mathrm{~s}$ character and has an ionization potential of $539.8 \mathrm{eV}$ [10]. In the outer-valence energy region, an overview of absolute absorption cross sections is given in Refs. [11-13], showing a complex structure of Rydberg series excitations for all valence orbitals.

Whereas processes with electron emission (or ion formation) are relatively easy to investigate by guiding the charged particles with suitable electric or magnetic fields to a detector, neutral fragments are much more difficult to detect. If these fragments are excited, however, fluorescence spectrometry can be used to detect their relaxation. This technique is particularly powerful when combined with excitation by monochromatized synchrotron radiation. This enables one to extract absolute and, if dispersed fluorescence is measured, even final dissociation-state selective cross sections [14-21].

Reference [22] gives an overview of energetic formation limits for excited fragments after excitation of water molecules. These thermodynamical limits can be calculated as the sum of the dissociation energy and the respective energy stored in the excited fragment. Below and slightly above the first ionization threshold the only observable fluorescence stems from $\mathrm{OH}^{*}$ transitions in the ultraviolet $(\mathrm{A} \rightarrow \mathrm{X}, \mathrm{C} \rightarrow \mathrm{X}, \mathrm{C} \rightarrow \mathrm{A})$ and visible $(\mathrm{B} \rightarrow \mathrm{A})$ range and from $\mathrm{H}_{2} \mathrm{O}^{+}(\mathrm{A} \rightarrow \mathrm{X})$ in the visible range [22]. The onset of fluorescence from neutral hydrogen 
atoms at $15.3 \mathrm{eV}$ is the sum of the $\mathrm{OH}+\mathrm{H}$ dissociation energy $(5.1 \mathrm{eV})$ and the energy of the lowest electronic excitation of ground state hydrogen, which corresponds to the Ly transition from $n=2$ to $n=1$ at $10.2 \mathrm{eV}$ (corresponding to $121.57 \mathrm{~nm}$ ). An overview of thermodynamical production limits of excited hydrogen after dissociation following photoexcitation and subsequent fluorescence decays by Lyman or Balmer emission is given in Table I.

Fluorescence emission from neutral hydrogen after photoexcitation of water molecules was also subject of several earlier investigations. Using synchrotron radiation, the absolute emission cross sections of $\mathrm{Ly}_{\alpha}$ fluorescence in the valence excitation energy range from 15 to $40 \mathrm{eV}$ were determined already more than 30 years ago [23]. Emission spectra and emission cross sections of $\mathrm{Ly}_{\alpha}$ and the first three Balmer transitions $\left(\mathrm{H}_{\alpha}, \mathrm{H}_{\beta}, \mathrm{H}_{\gamma}\right)$ for some excitingphoton energies were measured in early experiments $[24,25]$ in the excitation range from 15 to $22 \mathrm{eV}$, and a discussion of dissociation processes leading to Lyman and Balmer series emission is given. No formation of excited hydrogen fragments for exciting-photon energies above $22 \mathrm{eV}$ was observed in these early experiments. More recently, Ly $\mathrm{y}_{\alpha}$ emission on a relative scale was reported up to $60 \mathrm{eV}$ [26] and the resonances leading to the formation of excited hydrogen were discussed [27]. Relative emission functions and absolute cross sections are also available for the Balmer series up to $40 \mathrm{eV}[22,28,29]$.

TABLE I. Thermodynamical production limits $L$ of excited hydrogen fragments after dissociation of water molecules into $\mathrm{OH}\left(\mathrm{X}^{2} \Pi\right)+\mathrm{H}(n)$, the denotation $\mathrm{Ly}_{x}, \mathrm{H}_{x}$ of the subsequent Lyman and Balmer decays, and the corresponding fluorescence wavelengths $\lambda_{f l}$.

\begin{tabular}{lrllll}
\hline \hline & \multicolumn{3}{c}{ Lyman } & \multicolumn{2}{c}{ Balmer } \\
& $L(\mathrm{eV})$ & $\mathrm{Ly}_{x}$ & $\lambda_{f l}(\mathrm{~nm})$ & $\mathrm{H}_{x}$ & $\lambda_{f l}(\mathrm{~nm})$ \\
\hline 1 & 5.1 & - & - & - & - \\
2 & 15.3 & $\mathrm{Ly}_{\alpha}$ & 121.6 & - & - \\
3 & 17.2 & $\mathrm{Ly}_{\beta}$ & 102.6 & $\mathrm{H}_{\alpha}$ & 656.3 \\
4 & 17.9 & $\mathrm{Ly}_{\gamma}$ & 97.3 & $\mathrm{H}_{\beta}$ & 486.1 \\
5 & 18.2 & $\mathrm{Ly}_{\delta}$ & 95.0 & $\mathrm{H}_{\gamma}$ & 434.1 \\
6 & 18.3 & $\mathrm{Ly}_{\varepsilon}$ & 93.8 & $\mathrm{H}_{\delta}$ & 410.2 \\
$\infty$ & 18.7 & - & 91.2 & - & 364.6 \\
\hline \hline
\end{tabular}


In the exciting-photon energy region of core excitations, dissociation processes following Auger decays and leading to fluorescence are discussed in detail in Ref. [30]. There the fluorescence excitation function was measured for $\mathrm{Ly}_{\alpha}$ and some emission features of $\mathrm{OH}$, $\mathrm{OH}^{+}, \mathrm{O}$, and $\mathrm{O}^{+}$. It was found to resemble the general molecular excitation pattern, mirroring the unoccupied orbital structure of the water molecule with changing intensity ratios for certain excitations. By detecting the Balmer series $(\alpha-\delta)$ emission functions, the production functions of higher excited hydrogen atoms $(n \geq 3)$ have been determined [20]. For the molecular resonances $4 \mathrm{a}_{1}$ and $2 \mathrm{~b}_{2}$ it has been suggested that the main channel for production of excited hydrogen dissociation fragments is $\mathrm{H}_{2} \mathrm{O}^{+} \rightarrow \mathrm{H}(n \leq 3)+\mathrm{OH}^{+}$, where the initial water cation is formed by an Auger decay of the resonance. For higher, Rydberg-like excitations the Rydberg character was found to be conserved during the (resonant) Auger decay and the dissociation, leading to high- $n$ excited hydrogen fragments.

These experiments, however, and also those carried out in the valence excitation range, do not provide the complete information about the production of excited hydrogen atoms. For all $n \geq 3$ states the branching ratios for the different decay paths have not been experimentally determined, because only the Balmer series was observed. Measuring the Lyman series is experimentally difficult, because there is no transparent material in this photon energy region (except for $\mathrm{Ly}_{\alpha}$ ), requiring differential pumping stages. In the present work we will add experimental information about Lyman series emission cross sections. Using dispersed photon-induced fluorescence spectrometry [31], absolute fluorescence emission cross sections not only for the $\operatorname{Ly}_{\alpha}$ emission but for the Lyman series $(\alpha-\delta)$ lines in the valence excitation range are determined. The Lyman/Balmer branching ratios are computed and compared to theory. For the same lines relative emission functions after inner-shell excitation of water close to the $\mathrm{O}$ 1s-electron ionization threshold are given.

\section{EXPERIMENT}

Owing to the large difference between the exciting-photon energy ranges for valence and inner-shell excitations, two experiments were performed separately. Both of them were carried out with an established set-up for photon-induced fluorescence spectrometry (PIFS) [31], using a commercial McPherson $1 \mathrm{~m}$ normal incidence spectrometer, equipped with a gold-coated 1200 lines/mm grating to disperse the fluorescence. In the low energy 
region, the measurements were performed at the $10 \mathrm{~m}$ NIM beamline of undulator U125-2 of the Helmholtz-Zentrum Berlin (BESSY II) [32]. Two energy regions of the linear horizontally polarized synchrotron radiation were scanned by varying the photon energy stepwise: between $15 \mathrm{eV}$ and $22 \mathrm{eV}$ in $25 \mathrm{meV}$ steps in first order of the beamline monochromator and between $21 \mathrm{eV}$ and $34 \mathrm{eV}$ in $100 \mathrm{meV}$ steps in second order, to optimize the photon flux in the respective energy region. With an exit slit width of $200 \mu \mathrm{m}$ a photon bandwidth of $20 \mathrm{meV}$ was achieved at $19 \mathrm{eV}$ in first order and at $25 \mathrm{eV}$ in second order. A target cell with pinholes for the synchrotron beam and a $2 \mathrm{~mm}$ slit towards the fluorescence monochromator was used. Inside the target cell a static pressure of 0.2 mbar was maintained using an inlet valve with a reservoir pressure of about 32 mbar (vapor pressure of water at about $25^{\circ} \mathrm{C}$ ). The water was degassed by repeated freeze and thaw cycles.

In the high exciting-photon energy region, the measurements were performed at the beamline P04 of the PETRA III storage ring at DESY, Hamburg. The energy range 533 $542 \mathrm{eV}$ was scanned in $100 \mathrm{meV}$ steps with an exit slit width of $1500 \mu \mathrm{m}$, resulting in $100 \mathrm{meV}$ bandwidth. Here, no target cell was used but a gas jet created by supersonic expansion of water vapor at about $50^{\circ} \mathrm{C}$ through a nozzle of $150 \mu \mathrm{m}$ diameter. The expansion chamber was separated from the interaction chamber by a $1 \mathrm{~mm}$ skimmer.

In both experiments the dispersed fluorescence photons were detected with an "open-face" stack of two micro-channel plates (MCPs) without photocathode. An operating voltage of $-1150 \mathrm{~V}$ was applied to the front of each MCP with respect to its rear side. Additionally, a $-190 \mathrm{~V}$ voltage between the anode and the rear side of the second MCP and a $-170 \mathrm{~V}$ voltage between both MCPs were applied, resulting in a potential of $-2660 \mathrm{~V}$ at the front of the first MCP with respect to the anode, which was kept at ground potential. By that, electrons were rejected from reaching the MCP. Ions were rejected by a mesh in front of the first MCP, set to $+200 \mathrm{~V}$. A position sensitive wedge and strip anode behind the second MCP was used to detect the dispersed fluorescence position resolved.

\section{RESULTS AND DISCUSSION}

The absolute emission cross section of $\mathrm{Ly}_{\alpha}$ fluorescence as function of the exciting-photon energy is shown in Fig. 1. Data shown as black squares were obtained in first order of the beamline monochromator and are normalized to the photon flux (see Sec. II). They are 


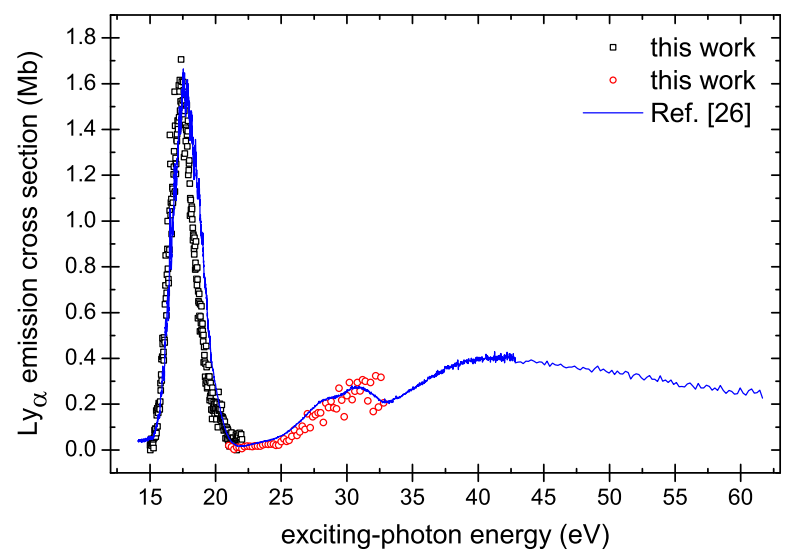

FIG. 1. (Color online) Absolute $\mathrm{Ly}_{\alpha}$ emission cross sections after excitation of water molecules with synchrotron radiation as function of the exciting-photon energy. Black squares: data measured in this work in first order of the beamline monochromator, normalized to the photon flux and calibrated to reference [23]. Red circles: measured in this work in second order of the beamline monochromator, normalized to the photon flux. Blue line: data from Ref. [26].

calibrated to the known cross section of Ref. [23] at an exciting-photon energy of $17.5 \mathrm{eV}$. Using the same procedure, the data from Ref. [26] were absolutely calibrated up to $60 \mathrm{eV}$. In turn, data shown as red circles, which were obtained in second order of the beamline monochromator up to $33 \mathrm{eV}$, are calibrated to the value of the curve of Ref. [26] at an exciting photon-energy of $31 \mathrm{eV}$. Above $33 \mathrm{eV}$, no reasonable results were obtained in the present experiment owing to extremely weak photon flux. From approximately $28 \mathrm{eV}$ on, the beamline intensity starts to decrease substantially and statistics become worse. However, within the uncertainty (discussed below), our relative cross sections agree with Ref. [26].

A. Exciting-photon energy range between $15 \mathrm{eV}$ and $22 \mathrm{eV}$ : dissociation of $1 b_{2}^{-1}$ states into $\mathbf{O H}\left(\mathrm{X}^{2} \Pi\right)+\mathbf{H}(n)$ fragment pairs

Figure 2(a) shows the absolute Lyman series fluorescence emission cross sections as functions of the exciting-photon energy emitted from neutral excited hydrogen dissociation fragments after valence electron excitations of water with energies between $15 \mathrm{eV}$ and $22 \mathrm{eV}$. The thermodynamical limits for the dissociation of water into $\mathrm{OH}\left(\mathrm{X}^{2} \Pi\right)+\mathrm{H}(n)$ fragment pairs are listed in Table I. All observed Lyman lines start emitting at their respective ther- 


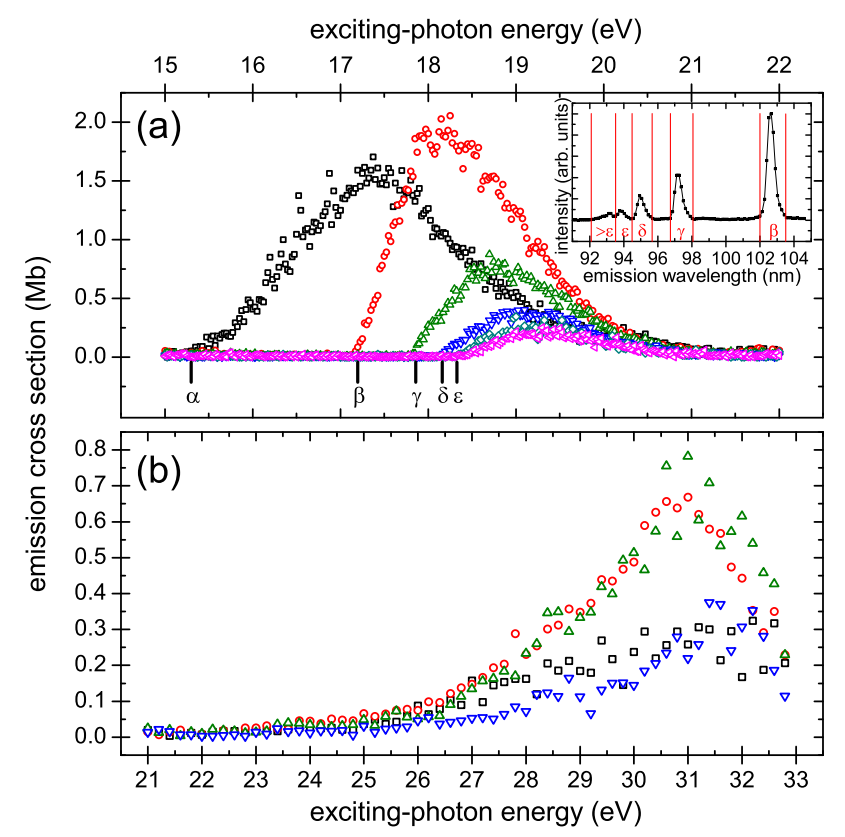

FIG. 2. (Color online) Lyman series emission after valence excitation of gaseous water with synchrotron radiation. (a) Absolute cross sections for emission after excitation into $1 b_{2}^{-1}$ states, measured in first order of the beamline monochromator. Black squares: $\mathrm{Ly}_{\alpha}$, red circles: $\mathrm{Ly}_{\beta}$, green triangles: $\mathrm{Ly}_{\gamma}$, blue triangles: $\mathrm{Ly}_{\delta}$, cyan diamonds: $\mathrm{Ly}_{\varepsilon}$, magenta triangles: $\mathrm{Ly}_{>\varepsilon}$. Literature values of thermodynamical onsets are indicated by black bars [25]. The inset shows a cutout of the spectrally resolved fluorescence (without $\mathrm{Ly}_{\alpha}$ at $121.6 \mathrm{~nm}$ ). The Lyman transitions contributing to the cross section curves are labelled. In " $>\varepsilon$ ", all unresolved transitions higher than $\mathrm{Ly}_{\varepsilon}$ are integrated. (b) Absolute cross sections for emission after excitation into doubly excited and $2 \mathrm{a}_{1}^{-1}$ states, measured in second order of the beamline monochromator.

modynamical limits for the formation of the corresponding excited fragment. The observed energetic onset of the $\mathrm{Ly}_{\alpha}$ line agrees with observations in previous publications [22, 25] and the observed onsets for $\mathrm{Ly}_{\beta}$ and $\mathrm{Ly}_{\gamma}$ agree with the observed onsets of $\mathrm{H}_{\alpha}$ and $\mathrm{H}_{\beta}$, respectively [22], as these lines are branched transitions from the same initial states. Cross sections for the higher members of the Lyman series as a function of the exciting-photon energy have not been published yet.

The simultaneous observation of the $\mathrm{Ly}_{\alpha}$ line together with the higher members of the Lyman series in the dispersed fluorescence spectra enabled a determination of the absolute emission cross sections of the higher Lyman series lines (Fig. 2(a)), calibrated to the known 
$\mathrm{Ly}_{\alpha}$ emission cross section [23-25]. The uncertainty of the literature values is given as $30 \%$ [23]. The uncertainty caused by the spatial response of different detector areas for position sensitive detection of the dispersed fluorescence was determined by measuring the intensity of the same line at different positions and is $20 \%$. No correction of the spectral detection efficiencies was attempted here, as the quantum efficiency of bare MCPs is assumed to be constant within the Lyman series range [33, 34]. Considering Gaussian uncertainty propagation, the total uncertainty for the absolute cross section values is therefore in the range of $40 \%$. The relative uncertainties within one dispersed fluorescence emission function are essentially the statistical uncertainties of the measurement and are typically at $5 \%$ in the range from 15 to $22 \mathrm{eV}$ and at $20 \%$ above $22 \mathrm{eV}$. The inset of Fig. 2(a) shows a cutout of a typical fluorescence spectrum. Integration of all Lyman transitions yields a maximum Lyman emission cross section of about $3.7 \mathrm{Mb}$ at an exciting-photon energy of $18.5 \mathrm{eV}$. By comparing it with the known absorption cross section in the respective exciting-photon energy range (about $20 \mathrm{Mb},[11]$ ) we find that about $20 \%$ of the absorption probability results in the formation of an excited hydrogen dissociation fragment.

The maximum values of the determined absolute cross sections of the Lyman lines are compared with those of the corresponding Balmer lines [29] and the intensity ratio

$$
R_{\sigma}=\frac{\sigma_{\mathrm{Lyman}}}{\sigma_{\mathrm{Lyman}}+\sigma_{\mathrm{Balmer}}}=\frac{\sigma_{\mathrm{Ly}}}{\sigma_{\mathrm{Ly}}+\sigma_{\mathrm{H}}}
$$

is calculated and listed in Table II. From theory, for a given $n$ the branching ratio $R_{p, n}$ can be computed from the probabilities for the respective Lyman and Balmer transitions [35]:

$$
R_{p, n}=\frac{p_{\text {Lyman }}}{p_{\text {Lyman }}+p_{\text {Balmer }}}=\frac{p_{\text {Ly }}}{p_{\text {Ly }}+p_{\mathrm{H}}}
$$

It should be noted that the $\mathrm{Ly}_{\alpha}$ emission cross section function in the exciting-photon energy range from its onset to about $17.2 \mathrm{eV}$ represents the formation probability of $\mathrm{H}(2 \mathrm{p})$ excited fragments from the dissociating excitations of $\mathrm{H}_{2} \mathrm{O}$ via the dissociation channel $\mathrm{OH}\left(\mathrm{X}^{2} \Pi\right)+\mathrm{H}(n=2)$ alone. Above $17.2 \mathrm{eV}$, the measured cross sections are not equivalent to the formation probability of the corresponding excited hydrogen fragment. For all $n \geq 3$, fluorescence cascades are possible, populating states with lower $n$.

Additionally, the oscillator strengths functions for the Lyman emission $\left(\frac{\mathrm{d} f}{\mathrm{~d} E}\right)_{e m}(E)$ can be calculated analogous to Ref. [29] from the absolute emission cross section $\sigma_{e m}(E)$ by

$$
\left(\frac{\mathrm{d} f}{\mathrm{~d} E}\right)_{e m}(E)=\frac{\sigma_{e m}(E)}{4 \pi^{2} \alpha a_{0}^{2} R_{y}},
$$


with the fine structure constant $\alpha$, the Bohr radius $a_{0}$, and the Rydberg energy $R_{y}$. Table II lists the oscillator strengths for the excitation energies $E_{\text {peak }}$ with maximum emission cross section.

TABLE II. Maximum emission cross sections of the Lyman transitions $\sigma_{\text {Ly }}$ and determined branching ratios $R$ of Lyman and Balmer transitions. Also the oscillator strengths at the energies of maximum emission cross section $E_{\text {peak }}$ are given.

\begin{tabular}{cccccccc}
\hline \hline$n$ & Decay & $\begin{array}{c}\sigma_{\mathrm{Ly}} \\
(\mathrm{Mb})\end{array}$ & $\begin{array}{c}\sigma_{\mathrm{H}}[29] \\
(\mathrm{Mb})\end{array}$ & $\begin{array}{c}R_{\sigma} \\
\text { Exp. }\end{array}$ & $\begin{array}{c}R_{p, n} \\
\text { Theo. }[35]\end{array}$ & $\begin{array}{c}E_{\text {peak }} \\
(\mathrm{eV})\end{array}$ & $\begin{array}{c}(\mathrm{d} f / \mathrm{d} E)_{e m} \text { at } E_{\text {peak }} \\
\left(10^{-4} \mathrm{eV}\right)\end{array}$ \\
\hline 2 & $\mathrm{Ly}_{\alpha} /-$ & $1.5 \pm 0.8$ & - & 1 & 1 & 17.4 & 137 \\
3 & $\mathrm{Ly}_{\beta} / \mathrm{H}_{\alpha}$ & $2.0 \pm 1.0$ & 0.27 & $0.88 \pm 0.10$ & 0.89 & 18.2 & 178 \\
4 & $\mathrm{Ly}_{\gamma} / \mathrm{H}_{\beta}$ & $0.8 \pm 0.4$ & 0.06 & $0.93 \pm 0.07$ & 0.88 & 18.7 & 68 \\
5 & $\mathrm{Ly}_{\delta} / \mathrm{H}_{\gamma}$ & $0.4 \pm 0.2$ & 0.024 & $0.94 \pm 0.07$ & 0.88 & 19.2 & 34 \\
6 & $\mathrm{Ly}_{\varepsilon} / \mathrm{H}_{\delta}$ & $0.26 \pm 0.13$ & 0.012 & $0.95 \pm 0.05$ & 0.89 & 19.3 & 24 \\
\hline \hline
\end{tabular}

An assignment of the resonant excitations in this excitation energy range, leading to dissociation into excited neutral hydrogen fragments, was described in several previous publications. Excitations were attributed to $1 \mathrm{~b}_{2}^{-1} 4 \mathrm{sa}^{1}$ and $1 \mathrm{~b}_{2}^{-1} 5 \mathrm{sa}^{1}$ states in the range from 15 to $20 \mathrm{eV}[11,22]$. Recently, an alternative assignment to $1 b_{2}^{-1} n l a_{1}^{1}$ and $1 b_{2}^{-1} n l b_{2}^{1}$ was suggested [26].

B. Exciting-photon energy range between $22 \mathrm{eV}$ and $33 \mathrm{eV}$ : neutral dissociation of doubly excited states and dissociative ionization of $2 \mathrm{a}_{1}^{-1}$ states into $\mathrm{OH}^{+}(\mathrm{A})+\mathrm{e}^{-}+\mathbf{H}(n)$ fragments

Above $22 \mathrm{eV}$, the formation of excited hydrogen atoms has been investigated using $\mathrm{Ly}_{\alpha}$ and Balmer series cross section functions [26, 29] and electron energy loss spectroscopy in coincidence with $\mathrm{Ly}_{\alpha}$ detection [27]. The cross sections for all transitions show three pronounced features at about $24 \mathrm{eV}, 28 \mathrm{eV}$, and $31 \mathrm{eV}$. The energetic limit for dissociative ionization with the formation of excited hydrogen is at $28.2 \mathrm{eV}$. Therefore, the first two features were assigned to doubly excited states forming excited hydrogen atoms after neutral dissociation [26]. The feature at $31 \mathrm{eV}$ corresponds to single-hole one-electron $2 \mathrm{a}_{1}^{-1}$ states, 
decaying also by neutral dissociation or dissociative ionization into $\mathrm{OH}^{+}(\mathrm{A})+\mathrm{e}^{-}+\mathrm{H}(n)$. Absolute cross sections are available for the Balmer series for these excitations [29]. In the present experiment, statistics became worse in this range due to the decrease of the photon flux of the used beamline with increasing exciting-photon energy. Despite that, the general excitation structure of previous works was reproduced, as shown in Fig. 2(b). The doubly excited state at $24 \mathrm{eV}$ was reported to be weak compared to those at higher exciting-photon energies and can therefore not clearly be identified here. However, the intensity between $26 \mathrm{eV}$ and $33 \mathrm{eV}$ is composed of two maxima and the intensity ratio between the double excitation at $28 \mathrm{eV}$ and the $2 \mathrm{a}_{1}^{-1}$ state at $31 \mathrm{eV}$ agrees reasonably with previous results $[26,29]$. Above $33 \mathrm{eV}$, the $\mathrm{Ly}_{\alpha}$ emission was interpreted to stem from direct dissociative ionization into $\mathrm{H}(2 \mathrm{p})+\mathrm{OH}^{+}(\mathrm{X})+\mathrm{e}^{-}$. A comparison with the shape of the Balmer series emission cross section curves in this exciting-photon energy range was interpreted as a preferred production of hydrogen fragments with $n=2$ compared to higher states $(n \geq 3)$ [26]. However, in the present work an intensity ratio of approximately two was found of both $\mathrm{Ly}_{\beta}$ and $\mathrm{Ly}_{\gamma}$ with respect to $\mathrm{Ly}_{\alpha}$ for the excitations at $28 \mathrm{eV}$ and $31 \mathrm{eV}$. Apparently, the production cross section of $\mathrm{H}(n=3,4)$ dissociation fragments is much higher than that of $\mathrm{H}(n=2)$ for these excitations. We therefore refine the interpretation in Ref. [26] of the signal above $33 \mathrm{eV}$ : The production rate of hydrogen fragments with $n=3,4$ with respect to $n=2$ is higher for the excitations at $28 \mathrm{eV}$ and $31 \mathrm{eV}$ compared to direct dissociative ionization above $33 \mathrm{eV}$.

\section{Exciting-photon energy range between $533 \mathrm{eV}$ and $542 \mathrm{eV}$ : inner-shell excita-} tion

The excitation of inner-shell electrons into unoccupied orbitals and their assignments were also subject of previous works [36, 37]. Fluorescence emission after excitation of water vapor in this energy range has already been investigated $[20,30,38]$. The relative emission excitation functions for $\mathrm{Ly}_{\alpha}$ and several $\mathrm{OH}$ and $\mathrm{O}$ fragment transitions were reported [30] as well as for the Balmer series [20]. The intensity ratios of low Lyman ( $\left.\mathrm{Ly}_{\alpha}\right)$ and Balmer $\left(\mathrm{H}_{\beta}\right)$ lines after different excitations were studied and discussed extensively and can be understood by energetic reasons after spectator Auger decays [20, 30, 39, 40]. To produce excited hydrogen fragments, the internal energy of the Auger final states of $\mathrm{H}_{2} \mathrm{O}^{+*}$ must be 


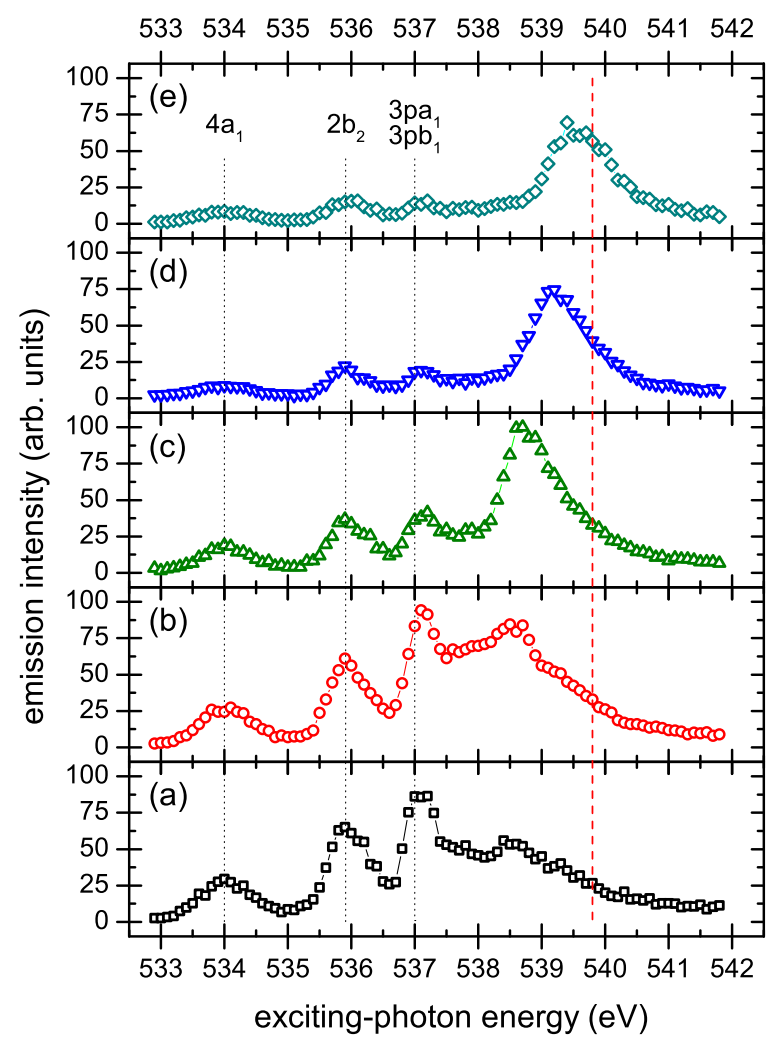

FIG. 3. (Color online) Relative Lyman series emission intensities as functions of the excitingphoton energy in the $\mathrm{H}_{2} \mathrm{O}$ inner-shell excitation range. (a) $\mathrm{Ly}_{\alpha}$, (b) $\mathrm{Ly}_{\beta}$, (c) $\mathrm{Ly}_{\gamma}$, (d) $\mathrm{Ly}_{\delta}$, (e) integrated intensity over all transitions higher than $\mathrm{Ly}_{\delta}$. All panels are set to the same relative scale. Black dotted lines indicate the first three molecular excitations [36]. The red dashed line at the exciting-energy of $539.8 \mathrm{eV}$ indicates the molecular inner-shell ionization threshold.

higher than the respective energy necessary for dissociation into $\mathrm{OH}^{+}+\mathrm{H}(n \geq 2)$, which is $16.5 \mathrm{eV}$ for $n=2$ and $17.5 \mathrm{eV}$ for $n=3[20,29]$. From the Auger spectra it can be deduced that for core excitations into the $4 \mathrm{a}_{1}, 2 \mathrm{~b}_{2}$, and $3 \mathrm{pa}_{1} / 3 \mathrm{pb}_{1}$ states only a small fraction of the Auger final states fulfill this condition $[39,40]$ and the intensity relations $3 \mathrm{pa}_{1} / 3 \mathrm{pb}_{1}>2 \mathrm{~b}_{2}>4 \mathrm{a}_{1}$ can be explained satisfactorily. However, the probability for the production of excited hydrogen fragments after these excitations is rather low. In contrast, excitation into Rydberg-like states close to the core ionization threshold yields a remarkable enhancement of Balmer emission compared to the molecular excitations [20].

The relative emission cross section of the Lyman series is shown in Fig. 3. The energies of 
the three lowest molecular excitations are labelled and indicated by black dotted lines. The red dashed line indicates the inner-shell ionization threshold. Panels (a)-(d) represent the spectrally resolved Lyman transitions $\mathrm{Ly}_{\alpha}-\mathrm{Ly}_{\delta}$. In panel (e), all unresolved transitions higher than $\mathrm{Ly}_{\delta}$ are integrated. Our results agree with the literature, the production of highly excited hydrogen fragments is enhanced in the energy range of Rydberg-like excitations. Intensity ratios in the $\mathrm{Ly}_{\beta}$ and $\mathrm{Ly}_{\gamma}$ excitation function agree with the corresponding curves for $\mathrm{H}_{\alpha}$ and $\mathrm{H}_{\beta}$ of Ref. [20]. For $\mathrm{H}(n \geq 4)$, Rydberg excitation leads to the highest production cross section in the core excitation range.

In Ref. [20], this significant increase in production probability of highly excited hydrogen atoms is explained by the different character of the Rydberg-like excitation. According to their analyses a highly excited molecular Rydberg state leaves the water molecule in a quasi doubly ionized state after a spectator Auger decay. Because of that, the Auger decay causes a collapse of the orbitals and enables a simultaneous shake up of the spectator electron. Subsequently, the systems dissociates into $\mathrm{OH}^{+}+\mathrm{H}^{+}$and the Rydberg electron attaches to one of the fragments while conserving its principal and angular quantum numbers. Thereby, the production of excited hydrogen becomes independent of the Auger final state. This model is supported by theoretical calculations of the overlap of the initially excited Rydberg states and final states of the shake up process. Using fluorescence spectroscopy, a similar behaviour of this "spectator dissociation" of Rydberg excitations was observed for innershell excitations of other molecules before [16-18]. Our results corroborate the suggested model and should stimulate further theoretical effort to determine quantitative production cross sections of excited fragments after core excitation of the water molecule.

\section{CONCLUSIONS}

To summarize, we measured relative and absolute emission cross sections for Lyman series emission after monochromatic valence and inner-shell excitation of gaseous water as functions of the exciting-photon energy. For emission in the range $15-33 \mathrm{eV}$ absolute emission cross sections were determined and oscillator strengths for the peak intensities were calculated. The results agree reasonably with complementary previous works on Lyman and Balmer emission. In the exciting-photon energy range $22-34 \mathrm{eV}$ previously reported superexcited states were corroborated. For inner-shell Rydberg excitations, we confirmed 
a spectator dissociation and gave relative cross sections for the Lyman series lines. Our results contribute to a completion of dissociation models and branching ratios in the decay processes of (photo)excited gaseous water molecules.

\section{ACKNOWLEDGMENTS}

This work was supported by the Hesse State Initiative for the Development of Scientific and Economic Excellence (LOEWE) in the LOEWE-Focus Electron Dynamics of Chiral Systems (ELCH) and by the BMBF grant under promotion code number 05K13RK1. We gratefully acknowledge HZB and DESY for beamtime allocation and the BESSY and DESY staff for support during the beamtimes. U.H. and M.F. acknowledge funding from DFG Forschergruppe 1789. We are grateful to N. Kouchi for providing us with his data that we show in Fig. 1.

[1] B. C. Garrett et al., Chem. Rev. 105, 355 (2005).

[2] B. Winter, Nucl. Instrum. Methods Phys. Res. A 601, 139 (2009).

[3] S. Thürmer, M. Ončák, N. Ottosson, R. Seidel, U. Hergenhahn, S. E. Bradforth, P. Slavíček, and B. Winter, Nat. Chem. 5, 590 (2013).

[4] L. S. Cederbaum, J. Zobeley, and F. Tarantelli, Phys. Rev. Lett. 79, 4778 (1997).

[5] S. D. Stoychev, A. I. Kuleff, and L. S. Cederbaum, J. Chem. Phys. 133, 154307 (2010).

[6] S. Barth, M. Ončák, V. Ulrich, M. Mucke, T. Lischke, P. Slavíček, and U. Hergenhahn, J. Phys. Chem. A 113, 13519 (2009).

[7] T. Jahnke et al., Nat. Phys. 6, 139 (2010).

[8] M. Mucke et al., Nat. Phys. 6, 143 (2010).

[9] M. S. Banna, B.H. McQuaide, R. Malutzki, and V. Schmidt, J. Chem. Phys. 84, 4739 (1986).

[10] R. Sankari et al., Chem. Phys. Lett. 380, 647 (2003).

[11] P. Gürtler, V. Saile, and E. E. Koch, Chem. Phys. Lett. 51, 386 (1977).

[12] W. F. Chan, G. Cooper, and C. E. Brion, Chem. Phys. 178, 387 (1993).

[13] G. N. Haddad and J. A. R. Samson, J. Chem. Phys. 84, 6623 (1986).

[14] H. Hertz, H. W. Jochims, H. Schenk, and W. Sroka, Chem. Phys. Lett. 29, 572 (1974). 
[15] E. D. Poliakoff, in Vacuum Ultraviolet Photoionization and Photodissociation of Molecules and Clusters, edited by N.C. Ng (World Scientific, New York, 1991).

[16] M. Ukai et al., Phys. Rev. Lett. 74, 239 (1995).

[17] H. Liebel, S. Lauer, F. Vollweiler, R. Müller-Albrecht, A. Ehresmann, H. Schmoranzer, G. Mentzel, K.-H. Schartner, O. Wilhelmi Phys. Lett. A 267, 357 (2000).

[18] A. Ehresmann, Ph. V. Demekhin, W. Kielich, I. Haar, M. A. Schlüter, V. L. Sukhorukov, and H. Schmoranzer, J. Phys. B 42, 165103 (2009).

[19] M. Glass-Maujean, Ch. Jungen, G. Reichardt, A. Balzer, H. Schmoranzer, A. Ehresmann, I. Haar, and P. Reiss, Phys. Rev. A 82, 062511 (2010).

[20] E. Melero García et al., Phys. Rev. Lett. 96, 063003 (2006).

[21] M. Meyer, S. Aloise, and A. N. Grum-Grzhimailo, Phys. Rev. Lett. 88, 223001 (2002).

[22] O. Dutuit, A. Tabche-Fouhaile, I. Nenner, H. Frohlich, and P. M. Guyon, J. Chem. Phys. 83, $584(1985)$.

[23] C. Y. R. Wu and D. L. Judge, J. Chem. Phys. 75, 172 (1981).

[24] J. E. Mentall, G. R. Möhlmann, and P. M. Guyon, J. Chem. Phys. 69, 3735 (1978).

[25] C. Y. R. Wu, E. Phillips, L. C. Lee, and D. L. Judge, J. Chem. Phys. 70, 601 (1979).

[26] M. Nakano, T. Odagiri, T. Tanabe, K. Funatsu, I. H. Suzuki, M. Kitajima, and N. Kouchi, J. Phys. B 43, 215206 (2010).

[27] T. Tsuchida et al., J. Phys. B 44, 175207 (2011).

[28] K. Mitsuke, J. Chem. Phys. 117, 8334 (2002).

[29] M. Kato et al., J. Phys. B. 37, 3127 (2004).

[30] A. Kivimäki et al., J. Phys. B 39, 1101 (2006).

[31] H. Schmoranzer, H. Liebel, F. Vollweiler, R. Müller-Albrecht, A. Ehresmann, K.-H. Schartner, and B. Zimmermann, Nucl. Instrum. Methods Phys. Res. A 467, 1526 (2001).

[32] G. Reichardt et al., Nucl. Instrum. Methods Phys. Res. A 467, 462 (2001).

[33] O. H. W. Siegmund, in Vacuum Ultraviolet Spectroscopy II, edited by J. A. R. Samson and D. L. Ederer (Academic Press, San Diego, 2000).

[34] Hamamatsu Photonics, in MCP Assembly technical information (Shimokanzo, Japan, 2006).

[35] H. A. Bethe and E. Salpeter, Quantum mechanics of one- and two-electron atoms (Plenum Publishing Corporation, New York, 1977).

[36] J. Schirmer et al., Phys. Rev. A 47, 1136 (1993). 
[37] K. Okada et al., Chem. Phys. Lett. 326, 314 (2000).

[38] A. Kivimäki et al., Phys. Rev. A 75, 014503 (2007).

[39] I. Hjelte et al., Chem. Phys. Lett. 334, 151 (2001).

[40] U. Hergenhahn et al., Chem. Phys. 289, 57 (2003). 